\title{
Estimation of Levels of Non-Enzymatic Antioxidants In Saliva of Chronic Periodontitis Patients with Type 2 Diabetes Mellitus Before And After Phase 1 Periodontal Therapy- A Prospective Follow Up Study
}

\author{
Dr Seba Abraham (Professor and HOD), Dr Aparna M Nair (Final Year MDS) \\ Dept Of Periodontics, PMS College Of Dental Science Trivandrum
}

\section{Introduction}

Periodontitis is one of the most common oral infections in human beings ${ }^{1}$ and it is also cited as one of the most significant reasons for tooth loss in adults. Investigations have displayed an increased risk of periodontitis in diabetic patients and this is because of the sheer number of cellular and molecular adaptations taking place in the periodontium as a sequel of prolonged hyperglycemia ${ }^{2}$. The imbalance between periodontal tissue destruction and repair concerning the host response to bacterial challenge results in periodontitis. Polymorphonuclear Neutrophils (PMNs) functions as the primary host defense against periodontal pathogens They produce and intensify the concentration of Reactive oxygen species (ROS) molecules which are adept in instigating periodontal tissue destruction ${ }^{3}$. Reactive oxygen species (ROS), including hydrogen peroxide, superoxide anion, and the hydroxyl radical, and reactive nitrogen species (RNS), including peroxynitrite, nitric oxide(NO), are common offshoots formed by the typical aerobic metabolism of oral cavity cells or by the inhalation of oxidizing agents in tobacco smoke and additional air pollutants. ${ }^{4-7}$

Diabetes mellitus (DM) includes a varied group of conditions characterized by altered glucose tolerance and damaged lipid and carbohydrate metabolism. Hyperglycemia is often referred to as a trademark of Diabetes mellitus ${ }^{8}$. Hyperglycemia prompts oxidative stress, which functions as an imbalance between the creation of reactive oxygen species or free radicals and the antioxidant defense mechanisms existing in biological systems. Oxidative stress is validated by an escalation in the reactive oxygen species levels and a fall in antioxidant defenses ${ }^{9}$. Several researchers, in their epidemiological, experimental, and clinical examination have testified that the pervasiveness and severity of periodontal diseases is considerably greater among diabetics than non-diabetics and their bidirectional relationship ${ }^{10-12}$. High glucose (HG) can induce the production of superoxide and hydrogen peroxide, the predecessors of reactive free radicals, which are competent enough to kindle the deterioration of antioxidant systems, by directly damaging many biomolecules, intensify lipid peroxidation and advance the insulin resistance in diabetes ${ }^{13-15}$. These free radicals are generated in organelles like cell membrane mitochondria, nucleus, lysosomes, peroxisomes, endoplasmic reticulum and cytoplasm ${ }^{16}$. Reactive free radicals are highly potent enough to create chemical modifications and thereby damage proteins, lipids, carbohydrates and nucleotides in the tissues ${ }^{17-18,14}$. The most common method for measuring free radical activity is to analyze the level of the end products of lipid peroxidation such as hydrogen peroxide, peroxynitrite, singlet oxygen peroxynitrous acid and peroxyl nitrite. Periodontitis has been acknowledged as the sixth complication of diabetes and its occurrence in type 2 diabetic patients is more than twice often been remarked that oxidative stress is a shared factor in periodontal disease and type 2 diabetes, and that the disparity in redox control resulting independently that of non-diabetic patients ${ }^{19-21}$. Diabetic patients demonstrate an increased severity of disease ${ }^{22,}{ }^{23}$. It has from these disease states acts synergistically and augments the biochemical and clinical sequence of these diseases in a bidirectional manner.

All organisms retain an antioxidant defense mechanism against the detrimental effects of ROS. Antioxidants are classified as 'those substances that are present at low concentrations, when related with those of an oxidisable substrate, which will considerably suspend or hinder oxidation of that substrate ${ }^{24}$. In normal physiology there is a vibrant balance between ROS activity and antioxidant defense capacity and when this balance changes in favor of ROS, either by a fall in antioxidant defense or a surge in ROS production or activity, it ensues in oxidative stress. Oxidative stress was described as 'a disruption in the pro-oxidant antioxidant equilibrium in favor of the former, leading to probable impairment ${ }^{25}$. The fluctuations in the host response as a result of several diseases can affect the supposed severity and prognosis of periodontitis.

Saliva contains a wide range of antioxidants including urate, ascorbate, reduced glutathione, oxidized glutathione and others ${ }^{26}$. These antioxidants work in unison, and the over-all antioxidant capacity may be the most pertinent factor for evaluating the defense ability ${ }^{27}$. It has been acknowledged that saliva functions as a faithful mirror of the body's health, as it consists of proteins, hormones, antibodies, and other molecules that are regularly analyzed in the regular blood tests to effectively screen health and disease ${ }^{28}$. 
The complex and multifaceted relationship between DM, periodontal disease, and salivary antioxidant status has not been effectively elucidated. Oxidative stress can be closely examined by inflammatory indicators like salivary antioxidants, reactive nitrogen species and reactive oxygen species. They play a very important role in the pathogenesis of periodontitis ${ }^{29}$. However, this mechanism is not coherently explicated in the case of diabetic patients with protracted periodontitis. We have theorized that the diabetic state would lessen the salivary antioxidants. There will also be an increase in lipid peroxidation products. Furthermore, this antioxidant deficiency may aid in analysing the crucial link between Type 2 Diabetes Mellitus and inflammatory periodontal disease. There is sufficient confirmation to hint that there is indeed a change in the levels of salivary antioxidants, ROS, RNS molecules in non-diabetics and chronic periodontitis. Nevertheless, there is a lack of coherent proof regarding the effect of SRP on salivary antioxidants (urate, ascorbate) and reactive nitrogen species (peroxynitrite) in Chronic periodontitis (CP) patients with DM.To the best of our knowledge there is no exhaustive study till date that compares the salivary levels of antioxidant and reactive nitrogen species (peroxynitrite) in CP patients with Type 2DM before and after Non-surgical periodontal therapy. Therefore, the present study is projected to consider the salivary antioxidant (urate ,ascorbate) level and peroxynitrite level in Chronic periodontitis patients with Type 2 DM before and after Scaling And Root planning.

\section{Study Population .}

\section{Material And Methods}

Subjects taken for the study comprised both male and female Type 2 controlled Diabetic patients who reported to the Department of periodontics, PMS College Of Dental Science. The subjects were drawn from a wide age spectrum of 30 to 60 years. Subjects were carefully chosen according to the specific criteria given by AAP and ADA. Patients were excluded if they suffered from any other systemic disease other than diabetes mellitus, uncontrolled diabetes mellitus, or had endured oral prophylaxis in the last 6 months, and those with aggressive periodontitis. Similarly, subjects with a history of antibiotic treatment within the time frame of the preceding three months or those with smoking history were also excluded. Subjects were enrolled from May 2014 to May 2015. The study was piloted in full agreement with the ethical principles, of the World Medical Association Declaration of Helsinki.The patients were given concise and brief information about the procedure before the initiation of the procedure and their written consent was obtained. Prior to treatment, patients were given guidelines on oral hygiene. The study was done in the morning between 9am to 11:00 am. Salivary, Ascorbate, peroxynitrite, and urate were analyzed spectrophotometrically before scaling \& root planning, 1 month, 3 months after scaling \& root planning.

\section{Results}

Table 1: Comparison of ascorbate in saliva of chronic periodontitis patients with Type 2 DM before $\&$ after phase 1 therapy

\begin{tabular}{|c|c|c|c|c|c|c|c|c|}
\hline Ascorbate & Mean & SD & $\mathrm{N}$ & F\# & $\mathrm{p}$ & Pair & $\begin{array}{l}\text { Mean } \\
\text { Diff. }\end{array}$ & $\mathrm{p} \$$ \\
\hline Baseline (A) & 0.20 & 0.05 & 30 & \multirow{3}{*}{$553.07 * *$} & \multirow{3}{*}{0.000} & A \& B & $0.07 *$ & 0.000 \\
\hline 1st month (B) & 0.27 & 0.04 & 30 & & & $A \& C$ & $0.12 *$ & 0.000 \\
\hline 3rd month (C) & 0.33 & 0.04 & 30 & & & $B \& C$ & $0.06^{*}$ & 0.000 \\
\hline
\end{tabular}

**: - Significant at 0.01 level, *: - Significant at 0.05 level

\#One-Way Repeated Measures ANOVA (Sphericity Assumed)

\$: Pair wise multiple comparison with Bonferroni Correction

Graph 1 : Comparison of ascorbate in saliva of chronic periodontitis patients with Type 2 DM before \& after phase 1 therapy

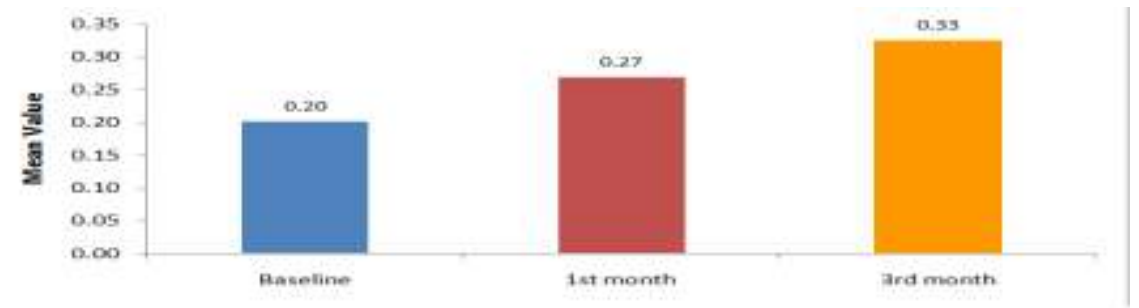

Mean ascorbate levels showed statistically significant $(\mathrm{P}<0.001)$ difference between before and after treatment. Average ascorbate was increased from $0.20 \mathrm{mg} / \mathrm{dl}$ to $0.27 \mathrm{mg} / \mathrm{dl}$ after treatment in first month follow up which was again increased to $0.33 \mathrm{mg} / \mathrm{dl}$ in the 3 month follow up

DOI: $10.9790 / 0853-1508077177 \quad$ www.iosrjournals.org $\quad 72 \mid$ Page


Table 2: Comparison of urate in saliva of chronic periodontitis patients with Type 2 DM before $\&$ after phase 1 therapy

\begin{tabular}{|c|c|c|c|c|c|c|c|c|}
\hline Urate & Mean & SD & $\mathrm{N}$ & $\mathrm{F} \#$ & $\mathrm{P}$ & Pair & Mean Diff. & $\mathrm{p} \$$ \\
\hline Baseline (A) & 0.19 & 0.12 & 30 & \multirow{3}{*}{$169.45^{* *}$} & \multirow{3}{*}{0.000} & A \& B & $0.07 *$ & 0.000 \\
\hline 1st month (B) & 0.27 & 0.13 & 30 & & & $A \& C$ & $0.17 *$ & 0.000 \\
\hline 3rd month (C) & 0.36 & 0.11 & 30 & & & $B \& C$ & $0.09 *$ & 0.000 \\
\hline
\end{tabular}

**: - Significant at 0.01 level, *: - Significant at 0.05 level

\#One-Way Repeated Measures ANOVA (Sphericity Assumed)

\$: Pair wise multiple comparison with Bonferroni. Correction

Graph 2: Comparison of urate in saliva of chronic periodontitis patients with Type 2 DM before \& after phase 1

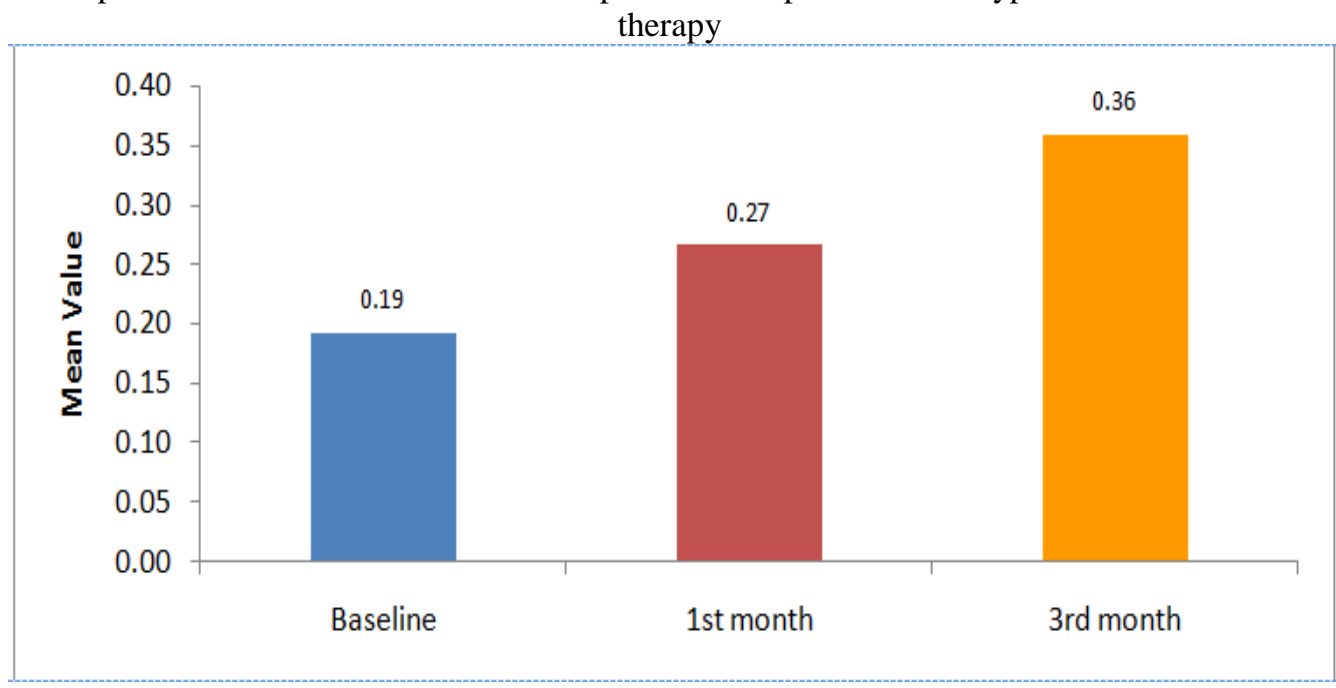

Mean urate showed significant $(\mathrm{P}<0.001)$ difference between before and after treatment. Average urate was increased from $0.19 \mathrm{mg} / \mathrm{dl}$ to $0.27 \mathrm{mg} / \mathrm{dl}$ after treatment in first month follow up which was again increased to $0.36 \mathrm{mg} / \mathrm{dl}$ in the 3 month follow up.

Table 3: Comparison of peroxynitrite in saliva of chronic periodontitis patients with Type 2 DM before \& after

\begin{tabular}{|c|c|c|c|c|c|c|c|c|}
\hline Peroxynitrite & Mean & SD & $\mathrm{N}$ & F\# & $\mathrm{p}$ & Pair & $\begin{array}{l}\text { Mean } \\
\text { Diff. }\end{array}$ & $\mathrm{p} \$$ \\
\hline Baseline (A) & 1019.6 & 161.9 & 30 & \multirow{3}{*}{$31.22 * *$} & \multirow{3}{*}{0.000} & A \& B & $52.07 *$ & 0.001 \\
\hline 1st month (B) & 967.6 & 130.1 & 30 & & & $\mathrm{~A} \& \mathrm{C}$ & $95.33^{*}$ & 0.000 \\
\hline 3rd month (C) & 924.3 & 120.4 & 30 & & & $\mathrm{~B} \& \mathrm{C}$ & $43.27 *$ & 0.000 \\
\hline
\end{tabular}

**: - Significant at 0.01 level, *: - Significant at 0.05 level

\#One-Way Repeated Measures ANOVA (Sphericity Assumed)

\$: Pair wise multiple comparison with Bonferroni. Correction

Graph 3 : Comparison of peroxynitrite in saliva of chronic periodontitis patients with Type 2 DM before \& after phase 1 therapy 


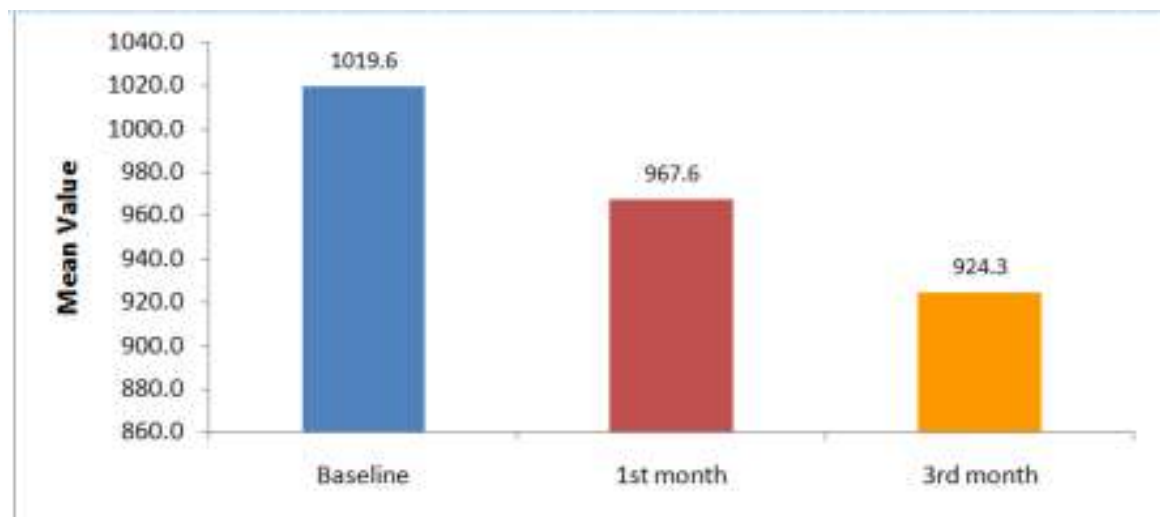

Mean peroxynitrite showed statistically significant $(\mathrm{P}<0.001)$ difference between before and after treatment. Average peroxynitrite was reduced from $1019.6 \mu \mathrm{g} / \mathrm{ml}$ to $967.6 \mu \mathrm{g} / \mathrm{ml}$ after treatment in first month follow up which was again reduced to $924.3 \mu \mathrm{g} / \mathrm{ml}$ in the 3 month follow up.

\section{Discussion}

Periodontal diseases can be cited as inflammatory diseases which possess multifactorial etiology. The inflammatory and immune reactions to the bacteria and viruses that affect the periodontal and associated tissues invariably influence the systemic circulation and eventually the peripheral systems of the body. This ensues in an intricate bi-directional succession of host - microbial interactions encompassing cellular and humoral aspects and networks of cytokines, chemokines, and growth factors. According to Haffajee et al the principal etiological cause for periodontal disease is specific which consists primarily of gram-negative anaerobic or facultative bacteria. However, the majority of periodontal tissue damage is instigated by an incongruous host reaction to those microorganisms and their products ${ }^{30}$. The timely detection of indicators of tissue destruction can thus be assimilated as a beneficial aid in the diagnosis of periodontal diseases.

Imbalance between reactive oxygen species and antioxidant defense has also been implicated as an etiologic factor for periodontal diseases. It may present itself as an escalation in oxidative stress, a reduction of total antioxidant capacity, or a fall in individual antioxidant level ${ }^{31}$. Chapple and Matthews implicated oxidative stress in the pathogenesis of periodontitis ${ }^{32}$.

Diabetes associated oxidative stress is often perceived a consequence in the production of free radicals or a reduced antioxidative defence capacity. A few studies ${ }^{33}, 34$ have assessed the salivary antioxidants in patients with diabetes before nonsurgical therapy, and contradictory reports were stated .So far, no studies have testified the effects of scaling and rootplanning on salivary levels of ascorbate, urate and peroxynitrite before and after phase 1 therapy in type 2 diabetic chronic periodontitis patients. Imbalances between the levels of free radicals,antioxidants and reactive oxygen species were proposed to play an imperative part in the inception and advance of various inflammatory oral diseases.

The present study was implemented to evaluate the salivary levels of urate, ascorbate and peroxynitrite at baseline, 1 month and 3 months after scaling and root planning. Thirty subjects between the age group of 30 and 60 were carefully chosen according to inclusion criteria. Salivary antioxidants ( urate, ascorbate) ,reactive nitrogen species ( peroxynitrite) and periodontal status were evaluated at baseline, 1 month and 3 month after Non-surgical periodontal therapy.

In the present study, there was a significant $(\mathrm{P}<0.001)$ reduction in $\mathrm{PI}$ and $\mathrm{OHI}$ score from baseline to 1 and 3 months. The mean OHI score at base line was 2.79 which were improved to 1.30 at 1 month and to 1 at 3 months after SRP. The mean PI at base line was 1.94 which reduced to 0.78 at 1 month and 0.62 at 3 months .This is in accordance with the study done by Nada Novokovic et al who testified a reduction in mean PI from 1.39 to 0.36 at 2 months after SPR in chronic periodontitis patients ${ }^{35}$.A significant improvement in OHI score and PI in our study could be ascribed to the level of oral hygiene maintained by the patients knowing that they are involved in a study and might have maintained good oral hygiene (Hawthorne effect) which could have affected the score of the indices.

There is also a significant $(\mathrm{P}<0.001)$ decrease in GI and BOP at 1 month and 3 month when compared to baseline. The mean GI at base line was 2 , which was reduced to 0.68 at 1 month and 0.03 at 3 month. BOP was obtained as 1.93 at baseline, which was reduced to 1.08 at 1 month and 0.33 at 3 months. This is comparable to study done by Nada Novokovic et al where he reported a decrease in GI in CP patients from 1.96 to 0.55 and reduction in BP from 1.81 to 0.43 at 2 months after SRP ${ }^{35}$. Non-surgical therapy led to a significant reduction in periodontal tissue inflammation which was identified by alterations in clinical factors in our study. SRP therapy is intended to reduce the disease triggering microbes allied with oral plaque. This may 
aid in diminishing the inflammatory indicators. Full mouth SRP might also remove the bulk of calculus and the related microbial flora.

The success of any periodontal treatment is determined by pocket depth reduction and gain in CAL. In the current study a statistically $(\mathrm{P}<0.001)$ substantial reduction in $\mathrm{PD}$ and gain in CAL was perceived when compared to the baseline at all-time intervals. However, an increase in PD was detected at 3 month when compared to 1 month in - patients; and this could be attributed to the improper oral hygiene maintenance. The mean pocket depth at base line was $6.1 \mathrm{~mm}$ and was lowered to $4.9 \mathrm{~mm}$ at 1 month and again increased to 5.1 $\mathrm{mm}$ at 3 month. The mean CAL at baseline was $6.2 \mathrm{~mm}$ and $5.9 \mathrm{~mm}$ and $5.7 \mathrm{~mm}$ at 1 month and 3 month consequently. This is in concordance with another study conducted by Nada Novokovic et al where he described $1.38 \mathrm{~mm}$ reduction in mean PD from base line and $0.02 \mathrm{~mm}$ gain in mean CAL compared to base line ${ }^{35}$. Thorough SRP has removed the bacterial burden which resulted in a discernible reduction in inflammation. Complete re-epethelization and healing by long junctional epithelium might have resulted in the reduction in PD and gain in CAL.

A decrease in the concentration of salivary ascorbate levels with an increase in severity of periodontal disease was detected through various investigative studies. The study done by Aziz A S et al reported an increase in plasma ascorbate level from( base line) $27.06 \mu \mathrm{M} / \mathrm{L}$ to $30.99 \mu \mathrm{M} / \mathrm{L}$ at 3 month after SRP ${ }^{36}$. In our study, a dedicated effort was undertaken to compare the salivary ascorbate and urate and peroxynitrite levels in diabetic CP patients before and after SRP was done. Since there is no other study till date, that compares the salivary ascorbate and urate levels in Diabetic CP patients before and after non surgical periodontal therapy, we will be comparing our results with non diabetic CP patients.

In the present research, the mean salivary ascorbate levels at baseline $(0.20 \mathrm{mg} / \mathrm{dl})$ was increased to $0.27 \mathrm{mg} / \mathrm{dl}$ at 1 month and to $0.36 \mathrm{mg} / \mathrm{dl}$ at 3 months after SRP. Thus, it demonstrates a statistically significant $(\mathrm{P}<0.001)$ difference between mean salivary ascorbate levels before and after treatment. The mean difference in the ascorbate levels between baseline and 1 month is 0.07 and between baseline and 3 month is 0.12 and between 1 month and 3 month is 0.06 which indicates a statistically significant $(P<0.001)$ increase in mean ascorbate level from base line to 1 month, base line to 3 month and 1 month to 3 month .

Our research augments the hypothesis that low ascorbate status in diabetes may be due to a higher turnover rate of ascorbic acid, with increased oxidation to its oxidized form dehydroascorbate. Another conceivable mechanism for the impaired ascorbate status is a competitive inhibition between glucose and ascorbic acid, which both share a close structural homology and possibly occupy common membrane transport sites. Yet another possible reason for the decreased ascorbate levels could be ascribed to lower consumption of fruits and vegetables rich in antioxidants or increased usage of antioxidants to counter oxidative stress ${ }^{37}$. Post-SRP therapy had a substantialprogress in salivary ascorbate levels signifying lowered oxidative stress and improved antioxidant status. In our study, salivary ascorbate level was lower in Diabetic chronic periodontitis at baseline as compared to 1 month and $3^{\text {rd }}$ month post operatively which agrees with other similar studies carried out in related area.

Vitamin $\mathrm{C}$ through its antioxidant action neutralizes oxidative stress and this may result in its reduction in plasma and saliva. It is therefore quite feasible that periodontitis causes lower salivary vitamin $\mathrm{C}$ through this mechanism. Human beings lack the ability to synthesise vitamin $\mathrm{C}$ due to mutation in the gene coding for $\mathrm{L}-$ glukonolactone oxidase, an enzyme that is mandatory for the biosynthesis of VitaminC via the glucuronic acid pathway. Thus, vitamin $\mathrm{C}$ is acquired through the diet. This vitamin is especially abundant in fresh fruit and in particular, citrus fruit and vegetables ${ }^{38}$.

In the present study, a significant reduction in concentration of salivary urate was observed at base line and a subsequent increase in urate levels at 1 and 3 months was noticed.

A previous study done by Scully et al also noted a reduction in mean salivary urate levels with increase in severity of periodontitis ${ }^{39}$. In our present study, there is a statistically significant increase in salivary urate level at all time intervals when compared to the base line. The mean urate level at baseline was $(0.19 \mathrm{mg} / \mathrm{dl})$ which increased to $0.27 \mathrm{mg} / \mathrm{dl}$ at 1 month and again increased to $0.36 \mathrm{mg} / \mathrm{dl}$ at 3 month after SRP showing statistically significant difference between before and after treatment. The mean difference in the urate levels between baseline and 1 month is $0.07 \mathrm{mg} / \mathrm{dl}$ and between baseline and 3 month is $0.17 \mathrm{mg} / \mathrm{dl}$ and between 1 month and 3 month is $0.09 \mathrm{mg} / \mathrm{dl}$ which indicates there is a statistically significant $(\mathrm{P}<0.001)$ increase in mean urate level from base line to 1 month, base line to 3 month and 1 month to 3 month subsequently

This is in agreement with previous studies done by NadNovokovic et $\mathrm{al}^{35}$, Pinar Gumus et al ${ }^{34}$, AditiMaduret et $\mathrm{al}^{31}$. SRP is aimed at reducing inflammation. The decreased pathogen load and local inflammation may help in upregulating salivary antioxidants and down regulating reactive nitrogen species as observed in our study. After scaling, when the inflammation had reduced, there was a subsequent increase in uric acid levels. 
This is the first study done to compare the levels of salivary peroxynitrite before and after scaling and root planing in diabetic chronic periodontitis patients. There is a statistically significant decrease in salivary peroxynitrite level before and after SRP at 1 month and 3 month. Mean salivary peroxynitrite level at baseline is $1019.6 \mu \mathrm{g} / \mathrm{ml}$ which is reduced to $967.6 \mu \mathrm{g} / \mathrm{ml}$ at 1 month and again reduced to $924.3 \mu \mathrm{g} / \mathrm{ml}$ at 3 month after SRP. The mean difference in the peroxynitrite levels between baseline and 1 month, base line and 3 month and 1 month and 3 month is $52.07 \mu \mathrm{g} / \mathrm{ml}, 95.33 \mu \mathrm{g} / \mathrm{ml}$ and $43.27 \mu \mathrm{g} / \mathrm{ml}$ respectively indicating a statistically significant $(\mathrm{P}<0.001)$ reduction in mean peroxynitrite level from base line to 1 month, base line to 3 month and 1 month to 3 month subsequently after phase 1 therapy. To the best of our knowledge no studies have evaluated the salivary peroxynitrite levels in chronic periodontitis patients with or without Diabetes. Hence we compare the results of our study to chronic obstructive pulmonary disease (COPD) and Rhuematoid arthritis (RH) which are inflammatory conditions similar to periodontitis. Kanasawa et al in 2003 assessed the imbalance between levels of nitrogen oxides and peroxynitrite inhibitory activity in chronic obstructive pulmonary disease in which they found increased peroxynitrite activity and increased levels of nitrogen oxides in induced sputum from patients with $\mathrm{COPD}^{40}$.

The study done by Kaur and Halliwelli described an elevated level of nitrotyrosine in serum, which is a marker of peroxynitrate production, from patients with RhumatoidArthritisis ${ }^{41}$. When compared with other similar studies, our research also noticed an elevated level of salivary peroxynitrite - a marker of oxidative stress in diabetic patients with periodontitis. There will be reduction in antioxidants and increase in Reactive nitrogen species resulting in chronic periodontitis. Phase 1 periodontal therapy causes reduction in periodontal pathogens and inflammation and intents to improve host defence by reinstating disturbed balance between antioxidant and reactive oxygen species. This points to an increase in the antioxidants and a decrease in reactive nitrogen species which are clearly evident from our study. Levels of peroxynitrite directly correlate with disease activity and hence can be considered as a marker for diabetic patients with periodontitis.

The collection of salivary components is a simple, non-invasive procedure that can be executed without a high level of technical proficiency. In addition, salivary samples can be effortlessly frozen and directed to laboratories for the examination of biomarkers. The analysis of saliva can also offer a cost-efficient method for the screening of large populations, and may denote asubstitutetechnique for patients in whom blood drawing is difficult, or when compliance is an issue. Saliva is predominantly useful for qualitative rather than quantitative diagnosis, which makes it essential for evaluating the efficiency during the maintenance phase. Stimulation of saliva results in elevated flow of GCF, thereby increasing salivary antioxidants level. Therefore, unstimulated whole-saliva samples were used for the present study.

One of the main drawbacks of our study is the lack of a definitive control group that would have helped us to conclude whether the levels of salivary antioxidants are categorically interrelated to the diabetic status, independently of the clinical periodontal situation.. This was a short term study and we recommend further longitudinal studies with large sample size to establish the findings of the present study and to appraise the efficaciousness of periodontal therapy in various systemic diseases associated with periodontitis.

\section{Reference}

[1]. Löe H. Periodontal disease. The sixth complication of diabetes mellitus. Diabetes Care 1993; 16:329-334.

[2]. Committee on Research, Science, and Therapy of the American Academy of Periodontology: position paper: diabetes and periodontal disease. J Periodontol. 1996;67:166-176

[3]. Salvi GE, Yalda B, Collins JG, et al. Inflammatory mediator response as a potential risk marker for periodontal diseases in insulindependent diabetes mellitus patients. J Periodontol 1997;68:127-135.

[4]. Minczykowski A, Woszczyk M, Szczepanik A,Lewandowski L, Wysocki H. Hydrogen peroxide and superoxide anion production by polymorphonuclearneutrophils in patients with chronic periapical granuloma,before and after surgical treatment. Clin Oral Investig 2001;5:6-10.

[5]. Waddington R, Moseley R, Embery G.Periodontal Disease Mechanisms: Reactive oxygen species: a potential role in the pathogenesis of periodontal diseases. Oral Diseases. 2000;6(3):138-51.

[6]. Paquette DW, Williams RC. Modulation of host inflammatory mediators as a treatment strategy for periodontal diseases. Periodontol 2000;24:239-252.

[7]. Daghigh F, Borghaei RC, Thornton RD, Bee JH. Human gingival fibroblasts produce nitric oxide in response to proinflammatory cytokines. J Periodontol2002;73:392-400.

[8]. Vernillo AT. Diabetes Mellitus: Relevance to dental treatment. Oral Surg Oral Med Oral Pathol Oral Radiol Endod. 2001;91:26370.

[9]. Bonnefont-Rousselot D. Glucose and reactive oxygen species. Curr Opin Clin Nutr Metab Care. 2002 Sep;5(5):561-8.

[10]. Stumvoli M, Goldstain B, Timon WH. Type 2 diabetes: Principles of pathogenesis and therapy. Lancet. 2005;65:333-46.

[11]. Waeber GP, Vollenweider PP. Prevention of type 2 diabetes: Where do we stand? Rev Med Suisse. 2007;3:291-5.

[12]. Lipinski B. Pathophysiology of oxidative stress in diabetes mellitus. J Diabetes Complications. 2001;15:203-10.

[13]. Nessar A. Advanced glycation end products: Role in pathology of diabetic complications. Diabetes Res Clin Pract. 2005;67:3-21.

[14]. Houstis N, Rosen ED, Lander ES. Reactive oxygen species have a causal role in multiple forms of insulin resistance. Nature. 2006;440:944-8

[15]. Valko M, Leibfritz D, Moncol J, Cronin MT, Mazur M, Telser J. Free radicals and antioxidants in normal physiological functions and human disease. Int J Biochem Cell Biol. 2007;39:44-84.

[16]. Valko M, Leibfritz D, Moncol J, Cronin MTD, Mazur M, Joshua T. Free radicals and antioxidants in normal physiological functions and human disease. Int J Biochem Cell Biol 2007; 44-84. 39. 
[17]. Brownlee M. Biochemistry and molecular cell biology of diabetic complications. Nature 2001;414:813-20.

[18]. Kiritoshi S,Nishikawa T, Sonoda K, Kukidome D, Senokuchi T, Matsuo T, et al. Reactive Oxygen Species from mitochondria induce Cyclooxygenase-2 gene expression in human mesangial cells: Potential role in diabetic nephropathy. Diabetes 2003;52:2570-7.

[19]. Loe H. Periodontal Disease. The sixth complication of diabetes mellitus. Diabetes Care 1993;16:329-34

[20]. Cohen DW, Friedman LA, Shapiro J, Kyle JC, Franklin S. Diabetes mellitus and Periodontal Disease: Two year longitudinal observations. J Periodontol 1970;41:709-12.

[21]. Nelson RG, Shlossman M, Budding LM, Pettitt DJ, Saad MF, Genco RJ, et al. Periodontal disease and NIDDM in Pima Indians. Diabetes Care 1990;13:836-40.

[22]. Tsai C, Hayes C, Taylor GW. Glycaemic control of type 2 diabetes and severe periodontal disease in the US adult population. Community Dent Oral Epidemiol 2002;30:182-92.

[23]. Tervonen T, Oliver RC. Long-term control of diabetes and periodontitis. J Clin Periodontol 1993;20:431-5.

[24]. Chapple IL. Reactive oxygen species and antioxidants in inflammatory diseases. J Clin Periodontol. 1997;24:287-96.

[25]. Sies, H. Oxidative Stress: From Basic Research to Clinical Application. The American Journal of Medicine.1991;91: 31-38.

[26]. Halliwell B. Reactive oxygen species in living systems: Source, biochemistry, and role in human disease. Am J Med. 1991;91:14$22 \mathrm{~s}$.

[27]. Brock GR, Butterworth CJ, Matthews JB, Chapple IL. Local and systemic total antioxidant capacity in periodontitis and health. J Clin Periodontol. 2004;31:515-21.

[28]. McDevitt JT. Saliva as the next best diagnostic tool. J Biochem 2006;45:23-5

[29]. Parveen Dahiya, Reet Kamal,Rajan Gupta, Rohit Bhardwaj, Karun Chaudhary, and Simerpreet KaurReactive oxygen species in periodontitisJ Indian Soc Periodontol. 2013;17(4): 411-416

[30]. Haffajee AD, Socransky SS. Microbial etiological agents of destructive periodontal diseases. Periodontol 2000. 1994;5:78-111.

[31]. Aditi Mathur, Lalit Mathur, Balaji Manohar, Rajesh Shankarapillai, Hemant Mathur . Effect Of Antioxidants On Progression Of Periodontal Disease .International Journal Of Dental Clinics 2011:3(4):13-15

[32]. Chapple ILC, Matthews JB . The role of reactive oxygen and antioxidant species in periodontal tissue destruction. Periodontol 2000.2007; 42: 1-73

[33]. Chapple ILC, Matthews JB. The role of reactive oxygen and antioxidant species in periodontal tissue destruction Periodontol 2000 .2007;43:160-232

[34]. Pinar Gumus,Nurcan Buduneli, Sxevki Cxetinkalp, Samuel I. Hawkins, Diane Renaud, Denis F. Kinane, and David A. Scott. Salivary Antioxidants in Patients With Type 1 or 2 Diabetes and Inflammatory Periodontal Disease: A Case-Control Study .J Periodontol 2009;80:1440-1446

[35]. Novakovic N,Sasa Cakic, Tatjana Todorovic, Biljana Andjelski Raicevic, Ivan Dozic, Vanja Petrovic, Neda Perunovic, Sanja Spadijer Gostovic, Jana Kadovic, Sretenovic, Emina Colak.Antioxidative Status of Saliva before and after Non-Surgical Periodontal Treatment. Srp Arh Celok Lek. 2013;141:163-168

[36]. Abdul Samad Aziz, Madhav Govind Kalekar Tabita Benjamin, Adinath Narayan Suryakar ,Shankargouda Patil , Mohammed Nadeem Ahmed BijleShort-Term Effectiveness of Scaling and Root Planning on Periodontal Parameters, Systemic Inflammatory, and Oxidative Stress Markers in Smokers with Chronic Periodontitis. Journal of International Oral Health .2015; 7(10): 18.

[37]. Ford S E et al. The metabolic syndrome and antioxidant concentrations. Diabetes .2003; 52, 2346-52.

[38]. Ray G, Husain S. A. Oxidants, antioxidants and carcinogensis. Indian J of Exp. Biol. Non 2002; 40 1213-32

[39]. Scully DV, Langley-Evans SC. Periodontal disease is associated with lower antioxidant capacity in whole saliva and evidence of increased protein oxidation. Clin Sci (Lond). 2003; 105:167-72

[40]. H Kanazawa, S Shiraishi, K Hirata, J YoshikawaImbalance between levels of nitrogen oxides and peroxynitrite inhibitory activity in chronic obstructive pulmonary disease Thorax .2003;58:106-109

[41]. 41 Kaur H, Halliwell B. Evidence for nitric oxide-mediated oxidative damage in chronic inflammation. Nitroty- rosine in serum and synovial fluid from rheumatoid patients. FEBS Lett 1994;350:9-1 